Control/Tracking Number: 2017-A-2552-ESHG

Activity: ESHG Abstract

Current Date/Time: 2/13/2017 3:30:23 PM

\title{
Genome wide association study identifies two novel loci associated with female stress and urgency urinary incontinence
}

Author Block: R. Cartwright ${ }^{1}$, M. Jarvelin ${ }^{1}$, P. Miotla ${ }^{2}$, V. Khullar ${ }^{1}$, P. Bennett ${ }^{1}$, A. Walley ${ }^{3}$, the IGNITE Consortium; ${ }^{1}$ Imperial College London, London, United Kingdom, ${ }^{2}$ University of Lublin, Lublin, Poland, ${ }^{3}$ St George's Medical School, London, United Kingdom.

\section{Abstract:}

Introduction: Stress and urgency incontinence are heritable, but no risk loci have been identified. We undertook a GWAS, using three European cohorts, followed by replication in six further studies, with supplementary transcriptomic analyses using human bladder biopsies.

Materials and Methods: Genotyping in discovery cohorts $(n=8,979)$ was conducted using lllumina arrays. Replication genotyping used competitive PCR $(n=4,069)$. Biopsies from women with urgency or stress incontinence were run on Affymetrix-U133 arrays.

Results: Discovery analyses identified five genome-wide significant loci. Two loci replicated: rs138724718 $\left(\mathrm{p}=3.39 \times 10^{-09}\right)$ and $\mathrm{rs34998271}\left(\mathrm{p}=1.70 \times 10^{-09}\right)$. In analysis of differential expression, the top-ranked process (GO:0003012, $p=7.5 \times 10^{-10}$ ), includes CHRM3 (fold difference:4.23, $p=0.0007$ ), which is the main drug target for urgency incontinence, $S U L F 2$ (fold difference:1.52, $p=0.005$ ) in the top locus from the discovery phase, and EDN1 (fold difference:-1.60, $\mathrm{p}=0.09$ ) in the top locus from the replication phase.

Conclusions: We identified two genetic variants strongly associated with urinary incontinence. The first, rs138724718, is situated near MARCO, with a role in host defense. The second, rs34998271, is situated near EDN1 a potent constrictor of smooth muscle, which was differentially expressed in bladder. This work highlights the myogenic and urotheliogenic mechanisms for incontinence, and suggests the potential of endothelin modulating drugs for urgency incontinence.

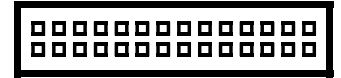

\begin{tabular}{|c|c|c|c|c|c|c|c|c|c|c|c|c|c|}
\hline \multirow[t]{2}{*}{ Chr } & \multirow[t]{2}{*}{$\begin{array}{l}\text { GRCh37 } \\
\text { Position }\end{array}$} & \multirow[t]{2}{*}{ SNP } & \multirow[t]{2}{*}{\begin{tabular}{|l|} 
Effect \\
Allele \\
\end{tabular}} & \multirow[t]{2}{*}{\begin{tabular}{|l|} 
Other \\
Allele \\
\end{tabular}} & \multirow[t]{2}{*}{ MAF } & \multirow[t]{2}{*}{ Phenotype } & \multicolumn{3}{|c|}{$\begin{array}{l}\text { Discovery Cohorts } \\
(\mathrm{n}=8,997)\end{array}$} & \multicolumn{3}{|c|}{$\begin{array}{l}\text { Replication Cohorts } \\
(\mathrm{n}=4,069)\end{array}$} & \multirow{2}{*}{$\begin{array}{l}\text { Overall } \\
\mathbf{p}\end{array}$} \\
\hline & & & & & & & OR & 95\%Cl & $p$ & $\overline{\mathrm{OR}}$ & $95 \% \mathrm{Cl}$ & $p$ & \\
\hline 20 & 46424160 & |rs139329202 & C & 9 & 0.01 & UUI & 8.50 & $\begin{array}{l}4.12- \\
17.55 \\
\end{array}$ & $8.07 \times 10^{-9}$ & 1.35 & $\begin{array}{l}0.82- \\
2.21\end{array}$ & 0.238 & $2.38 \times 10^{-05}$ \\
\hline 6 & |1430664 & |rs146033157 & a & $\mathrm{t}$ & 0.03 & UUI & 0.33 & $\begin{array}{l}0.23- \\
0.48 \\
\end{array}$ & $1.73 \times 10^{-8}$ & || 0.97 & $\begin{array}{l}0.35- \\
2.71\end{array}$ & 0.960 & n/a \\
\hline 14 & ||55489229 & $\|$ rs146757102 || & a & 9 & 0.05 & UUI & 0.45 & $\begin{array}{l}0.34- \\
0.60\end{array}$ & $1.95 \times 10^{-8}$ & $\mid 1.13$ & $\begin{array}{l}0.87- \\
1.45\end{array}$ & 0.360 & $5.12 \times 10^{-03}$ \\
\hline 7 & ||141328145 & |rs78851245 & $\mathrm{t}$ & C & 0.02 & Any UI & 3.22 & \begin{tabular}{|l}
$2.13-$ \\
4.86 \\
\end{tabular} & 2.9 & $\mid 1.46$ & $\begin{array}{l}1.00- \\
21.3 \\
\end{array}$ & 0.051 & $2.11 \times 10^{-07}$ \\
\hline 7 & ||34354797 & |rs78878767 & a & C & 0.01 & UUI & 4.26 & $\begin{array}{l}2.56- \\
7.10- \\
\end{array}$ & $3.04 \times 10^{-8}$ & || 0.86 & $\begin{array}{l}0.51- \\
1.43 \\
\end{array}$ & 0.556 & $2.11 \times 10^{-07}$ \\
\hline 3 & 55473083 & rs13059018 & c & 9 & 0.07 & SUI & 0.70 & \begin{tabular}{|l}
$0.61-$ \\
0.81 \\
\end{tabular} & $1.01 \times 10^{-7}$ & $\mid 1.14$ & $\begin{array}{l}1.00- \\
1.29 \\
\end{array}$ & 0.054 & $1.40 \times 10^{-06}$ \\
\hline 12 & ||11049362 & |rs201363123 & ag & a & 0.06 & Any UI & 0.65 & $\begin{array}{l}0.56- \\
0.76 \\
\end{array}$ & $1.03 \times 10^{-7}$ & $\mid 1.14$ & $1.00-$ & 0.053 & $5.43 \times 10^{-05}$ \\
\hline 1 & |154881110 & ||rs1218596 & $\mathrm{t}$ & C & 0.06 & Any UI & 0.64 & $\begin{array}{l}0.55- \\
0.75 \\
\end{array}$ & $1.04 \times 10^{-7}$ & || 0.95 & $\begin{array}{l}0.75- \\
1.20 \\
\end{array}$ & 0.681 & $4.49 \times 10^{-06}$ \\
\hline 11 & ||39642765 & |rs10768519 & a & c & 0.26 & UUI & 0.80 & $\begin{array}{l}0.74- \\
0.87\end{array}$ & $2.26 \times 10^{-7}$ & $\mid 1.00$ & $\begin{array}{l}0.85- \\
1.17 \\
\end{array}$ & 0.954 & $1.40 \times 10^{-04}$ \\
\hline 9 & ||105517298 & rs72738866 & $\mathrm{t}$ & C & 0.26 & SUI & 0.79 & \begin{tabular}{|l}
$0.71-$ \\
0.87 \\
\end{tabular} & $2.55 \times 10^{-7}$ & $\mid 1.01$ & $\begin{array}{l}0.89- \\
1.14 \\
\end{array}$ & 0.895 & $5.97 \times 10^{-05}$ \\
\hline 2 & ||119587824 & |rs138724718 & a & 9 & 0.02 & SUI & 1.85 & $\begin{array}{l}1.47- \\
2.35 \\
\end{array}$ & $2.89 \times 10^{-7}$ & $\mid 1.73$ & $\begin{array}{l}1.20- \\
2.48 \\
\end{array}$ & 0.003 & $3.39 \times 10^{-09}$ \\
\hline 6 & ||12533066 & |rs34998271 & a & 9 & 0.05 & UUI & 1.70 & $\begin{array}{l}1.40- \\
2.07\end{array}$ & $\begin{array}{l}4.97 \times 10^{-} \\
7\end{array}$ & |1.55 & $\begin{array}{l}1.20- \\
2.01\end{array}$ & 0.0008 & $1.70 \times 10^{-09}$ \\
\hline
\end{tabular}


Author Disclosure Information:

R. Cartwright: None. M. Jarvelin: None. P. Miotla: None. V. Khullar: None. P. Bennett: None. A. Walley: None. Topic (Complete): 03. Internal organs \& endocrinology (lung, kidney, liver, gastrointestinal)

Keyword (Complete): Incontinence

Presentation Preference (Complete): Oral preferred

Awards - Fellowships (Complete):

I apply for the Young Investigator Award: Yes

I am a: Post-doc researcher

Date of PhD/MD - dd.mm.yyyy: : 01.04.2016

Application for Fellowship: No

Application for Fellowship: No

Application for Fellowship: Yes

Status: Complete

ESHG Conference c/o Vienna Medical Academy

Alser Strasse 4, A-1090 Vienna, Austria

Tel: (+43/1) 40513 83-22

$\operatorname{Fax}(+43 / 1) 4078274$

Leave OASIS Feedback Leave OASIS Feedback

Powered by cOASIS, The Online Abstract Submission and Invitation System SM

(c) 1996 - 2017 CTI Meeting_Technology_ All rights reserved. 